\title{
Ambiances
}

anbiances Environnement sensible, architecture et espace urbain Comptes-rendus | 2014

\section{Ambiances et entre-deux}

\section{Pedro José García Sánchez}

\section{OpenEdition \\ Journals}

Édition électronique

URL : http://journals.openedition.org/ambiances/413

DOI : 10.4000/ambiances.413

ISSN : 2266-839X

\section{Éditeur :}

Direction Générale des Patrimoines - DAPA - MCC, UMR 1563 - Ambiances Architectures Urbanités (AAU)

\section{Référence électronique}

Pedro José García Sánchez, « Ambiances et entre-deux », Ambiances [En ligne], Comptes-rendus, mis en ligne le 10 février 2014, consulté le 22 septembre 2020. URL : http://journals.openedition.org/ ambiances/413; DOI : https://doi.org/10.4000/ambiances.413

Ce document a été généré automatiquement le 22 septembre 2020.

\section{(c) (i) () $\Theta$}

Ambiances is licensed under a Creative Commons Attribution-NonCommercial-NoDerivatives 4.0 International License. 


\title{
Ambiances et entre-deux
}

\author{
Pedro José García Sánchez
}

\section{De Grenoble à Montréal}

1 L'emprise du sensible sur l'espace et sur ses modes de partage a fait débattre ces dernières années d'un côté et de l'autre de l'Atlantique. Prenons comme exemple une prétention souvent avérée : le fait que, tout au plus, l'ambiance soit de l'esthétique. On peut même espérer encore moins que cela et amener la prétention jusqu'au bout: l'ambiance serait alors «du vent, du luxe, de l'hédonique et de l'accessoire» (Augoyard, 2011a). Par cette provocation, aucunement gratuite et délibérément paradoxale, Jean-François Augoyard inaugurait le $1^{\text {er }}$ Congrès International sur les Ambiances à Grenoble en 2008. Il y a eu certes ce geste surprenant mais révélateur d'un choix de méthode : l'astuce rhétorique consistant à « couper l'herbe sous le pied » des critiques pour mieux cerner l'essentiel. Mais il s'en est suivi également un magistral exercice intellectuel et de style destiné à montrer qu'il n'y a rien de mieux pour convaincre que de prendre au sérieux les critiques ayant pu éprouver le devenir objet $\mathrm{du}$ sujet "ambiances». Accordons enfin aussi toute son importance au fait que « l'essentiel » à Grenoble en 2008 était, tout simplement, l'invitation à vivre et à réaliser l'expérience à laquelle on nous avait conviés, expérience marquée d'abord, par sa sensibilité et sa mise en forme plurielles et, ensuite, par sa transmission et son intelligibilité distribuées.

2 Néanmoins, cette (re)présentation et son cadre montraient aussi autre chose : la portée, à la fois rétrospective, affirmative et programmatique d'une entrée en matière indéniablement pragmatiste et résolument interpellatrice à l'égard de l'aménagement urbain. L'expérience citadine n'aurait pas alors à renier son élan exploratoire ni à camoufler son côté expérimental (ce qui, au passage, ne peut que réjouir les architectes, les créateurs et les autres supporteurs d'une « culture du projet » - parfois trop sacralisée par ailleurs). Pour autant, la profondeur de champ théorique visée et la richesse des approches proposées ne laissaient pas non plus à désirer: depuis la neurophysiologie de la perception d'Alain Berthoz jusqu'à la pratique scénographique / 
scénologique de Guy-Claude François, en passant par la philosophie esthétique de Gernot Böhme et la pléthore des travaux des communicants de tous bords : acousticiens et anthropologues, paysagistes et historiens, urbanistes et chorégraphes, sociologues et architectes, designers et géographes... (Augoyard, 2011b).

Et « le social» dans tout cela ? Peut-être dans ce qui peut se révéler être sa plus juste place: habité par le sensible (plus qu'on ne le soupçonne), éprouvé par les inconséquences urbanistiques et les inégalités (pas seulement socioéconomiques) et cherchant pourtant ses marques dans l'entre-deux (plutôt que dans les identités insondables et les collectifs fourre-tout). C'est ainsi que résonne la maxime écologique que les ethnographes de Chicago ont tenu à nous apprendre ${ }^{1}$ : se rendre aux sens peut conduire aussi à rester attentifs aux interdépendances que les diverses formes de coprésence et d'engagement suscitent. L'espace peut ainsi ne pas être un réceptacle destiné à accueillir des êtres et/ou des objets. Le fait de parvenir à y susciter des aménités et à veiller aux atmosphères qu'elles produisent, peut alors être autre chose qu'une tâche éventuelle, mineure ou périphérique.

4 Dans le sillage de l'enjeu, certes, mais plus souvent sous le signe du défi, le cap était fixé et le ton donné pour les multiples activités (séminaires, ouvrages, rencontres...) qui depuis ont été produites ou chapotées par le Réseau International Ambiances (http:// www.ambiances.net/). La transformation du sujet (sensible) en objet (ambiance) est alors suggérée / opérée de façon stimulante, pratique, dense et innovante. En ce sens, les intitulés des congrès à Grenoble en septembre 2008, "Faire une ambiance », et de Montréal en septembre 2012, «Ambiances en acte(s) », n’ont pas été qu'allégoriques.

\section{Ambiances en acte(s) : l'urbanité des sens à l'épreuve du politique?}

Quid de l'essentiel quatre ans après à Montréal ? Jean-Paul Thibaud ouvrait les feux en évoquant des éléments qui tenaient du bilan aussi bien que du programme: un renouveau foisonnant, déplié et affirmatif de la notion d'usage ; une interrogation sur les possibilités ouvertes lorsqu'on s'aventure à dégager une pensée entre les langues, les langages et les professionnalités; un positionnement d'enquête à propos des transformations induites par ce qui pourtant demeure comme des incertitudes de l'inscription sociale / sociétale du sensible. David Howes, hôte "décontracté", a pratiqué ensuite la dérision comme forme de pédagogie: des consignes d'usage du patrimoine local (le sirop d'érable, la poutine québécoise ou encore le billet de cent dollars canadiens) devaient permettre, au moins, de maximiser l'expérience sensorielle de Montréal et, au plus, de comprendre en acte et in situ le sensory turn.

Le $2^{\text {nd }}$ Congrès International sur les Ambiances (Thibaud \& Siret, 2012) a été également l'occasion d'apercevoir l'hétérogénéité des entrées pratiquées et des outils proposés par les communicants: du geste comme moyen de transporter des sensibilités (Albertsen) au compositing (Meigneux) et à la vidéographie (Brayer) qui reconfigurent l'espace par des coupes mobiles incitant à d'autres montages; de la chaleur comme source esthétique et écologique (Ong) à la "kidkit» (boîte sonore interactive) permettant de «meubler " autrement l'attente des enfants dans des hôpitaux danois (Hojlund \& Kinch); des "breathing façades » qui font évoluer les ambiances thermiques en Egypte (Elghawaby) aux configurations végétales qui, en plus des corps, font respirer 
le voisinage (Balaÿ); du « pouvoir de l'étalagiste » dont le métier fait réfléchir à l'art de la suggestion (Monin) à la "tessiture d'un pas » (Bérubé), qui peut représenter bien plus qu'une partition dans la symphonie urbaine.

7 Le choix des conférencières invitées à Montréal (Linnaea Tillet et Elizabeth Diller), ainsi que leurs présentations, m'ont fait penser aux conséquences de la montée en généralité du thème " ambiances » chez les praticiens. S'il est vrai que celle-ci se traduit parfois en montée en puissance (des moyens et de visibilité, en particulier), elle semble également éprouver l'approche in situ privilégiée davantage par les chercheurs et par les créateurs. Car si, à juste titre, Tillet rappelait au début de sa conférence la dette des Nord-Américains à l'égard de William James qui, à son avis, leur a appris à répondre à "What is it?" par "What works! »2, comment alors faire pour que, par exemple, la fameuse High Line newyorkaise (ayant d'ailleurs contribué à la renommée de Diller) ne devienne pas qu'un modèle à reproduire, quel que soit le contexte ${ }^{3}$ ? Puis, le privilège accordé (et pas toujours justifié) aux récits-catalogues des parcours professionnels caractéristiques d'une "scene system architecturale mondialisée " ne serait-il pas également à interroger ?

Mirko Zardini, directeur du CCA, rappelle dans les actes du congrès les coordonnées d'un «nord» qui serait tout de même valable, aussi bien pour les uns que pour les autres: "Toward a sensorial urbanism» (2012). Signalons qu'une expression proche intitulait en 1996 la thèse doctorale de Grégoire Chelkoff: L'urbanité des sens. C'est l'occasion de souligner ce qu'une précaution sémantique en vigueur nous apprend: depuis les débats suscités par l'article renommé de Louis Wirth (1938), l'« urbanism » (anglosaxon) déborde l'« urbanisme » (latin) par ses rebords expérientiels, que ceux-ci éprouvent nos modes de vie ou qu'ils permettent d'assumer la puissance sensibilisatrice (citadine et citoyenne) des ambiances urbaines. Ce débordement pourrait-il être néanmoins relativisé par une mise en perspective qui amènerait les perceptions professionnelles diverses à se frotter au point de pouvoir se croiser sans réticences? L'horizon d'une interdisciplinarité à l'œuvre s'en dégagerait ainsi. Ces questionnements sont nécessaires, me semble-t-il, en particulier dans une époque où, tout en affirmant faire le contraire, (1) bien des maitrises d'œuvre et d'ouvrage n'hésitent pas à instrumentaliser le dessin des espaces publics et les présupposés participatifs pour conforter autrement la ségrégation socio-spatiale ainsi que celle des usages et (2) le marketing urbain mercantilise à outrance le profilage des usagers pour des usages non précisément citadins ou citoyens.

9 L'affichant d'une manière plus ou moins directe, les enquêtes sur la portée critique et politique des ambiances ont eu certainement une place importante dans le congrès de Montréal. La façon dont le travail sur les ambiances ne se contente pas de décrire ou de conforter les sensibilités ambiantes ni leurs formes plus spatialisées de réalisation, apparaît comme un souci commun ${ }^{5}$. S'agissant tantôt d'interpeller ces sensibilités ambiantes, tantôt de les transformer, les esquisses de réponse à la question du «comment?" deviennent alors centrales. Ainsi, des narrativités agissant comme "passerelles plutôt que comme autorités» pouvant rendre «le monde urbain perfectible " (Thomas, 2012) et des «identités ambiantales " pouvant "renouveler le renouvellement urbain» (Hollard, 2012) se trouvent parmi les pistes avancées. L'émergence d'une "climatologie politique " qui replacerait les émotions dans la différenciation des rythmes sociaux (Desroches, 2012) ou, encore, des «méthodologies de dépaysement » éprouvant les ambiances urbaines « à la fois comme tonalité affective 
et comme praxéologie ordinaire » (Pecqueux, 2012) ont, également, permis d'élargir le débat.

Néanmoins, force est de constater que certains auteurs ont du mal à affranchir leurs propos des automatismes dialectiques qui, dans l'histoire de la pensée moderne, ont souvent colonisé le positionnement critique et rigidifié la construction et la représentation des figures du politique. Les approches situationnelles, pragmatistes, phénoménologiques et micro-analytiques qui participent significativement à la fabrique des ambiances et à leur réflexivité, manqueraient-elles alors d'outils appropriés pour l'entreprise critique ainsi que pour le travail politique? Ou serait-ce plutôt que leurs porteurs demeurent parfois trop hésitants dans l'assomption des risques et des possibilités ouvertes par les perspectives que, pourtant, leurs propres travaux actualisent?

11 Ainsi, par exemple, tenir à l'identification contradictoire des "peurs positives ou négatives » (Desroches, op.cit.), à l'opposition entre subversion et "conservatisme politique» (Pecqueux, op.cit.) ou, encore, à une distinction segmentaire entre des " ambiances sidérantes" et des «ambiances aseptisées 》 (Bonicco-Donato, 2012), semblerait répondre au format que, depuis Hegel, le modèle dialectique impose à la critique. On pourrait cependant interroger les raisons qui poussent à enfermer l'analyse entre des options binaires qui, de gré ou de force (ou encore par inertie ou par osmose), finissent par privilégier un format qui corsette, me semble-t-il, l'ouverture critique.

at binaire et la grammaire cognitive où celui-ci s'inscrit, ont certes une prégnance communicationnelle souvent importante. L'on sait à quel point le fait "d'avoir le sens de la formule " peut octroyer une puissance qui, par exemple dans le contexte des arènes publiques, ne limiterait pas ses effets au champ rhétorique. C'est aussi pourquoi le format binaire et les mondes qu'il contribue à façonner sont souvent appropriés par des approches "prêt-à-porter» du politique. D'ailleurs, lorsque le devenir sensible du politique devient un enjeu sociétal, les dialectiques du genre "ami / ennemis", "avec/ contre», etc., ne manquent pas d'être mobilisées. Néanmoins, face au défi de celui qui «peut être là et ne pas être part» (Bordreuil, 2007), que faire de l'implicite cognitif qui accorde aux paires contradictoires la tâche de recouvrir (même "sensiblement») la globalité d'un phénomène? Par ailleurs, est-il juste de présupposer que dans les bords malléables de l'urbanité des sens, entre critique et politique, il y aurait une relation de cause à effet ? Comment approcher cet autre "entre-deux» dont les conséquences oscillent souvent entre l'implosion redoutée et l'explosion redoutable?

13 Face aux exigences du pluralisme démocratique, le sensible et le politique sembleraient s'interpeller mutuellement davantage. Et si, comme réflexe de méthode, au lieu d'entretenir une relation de nécessité avec l'univers dialectique et ses outils, on choisissait plutôt de partir de ses limites? On pourrait alors miser davantage sur la singularité à laquelle donne lieu le suivi rapproché d'un cours d'action, où qu'il passe et quel que soit le registre sensible qui l'éprouve. Ce suivi fera apparaître certainement des fragilités identitaires et interactionnelles, des répertoires d'engagements inachevés et des nuances catégorielles, bien plus que ce que les signifiants binaires et son fairesystème arrivent à (re) connaître ${ }^{6}$.

14 Alors «que faire du fond inextricablement politique des ambiances » (pour reprendre la stimulante question d'Anthony Pecqueux) ? Si la formule «ressentir c'est déjà agir » 
(Bonicco-Donato, op.cit.) est significative en termes pratiques, on peut questionner sa pertinence pragmatique - en particulier lorsqu'on pense que des exigences de publicité et de coordination sont censées accompagner l'activité pour prétendre échafauder autre chose que l'individualisme ambiant. Si le devenir politique du sensible commence par un positionnement délibéré (plutôt que délibérant), n'allons pas non plus trop vite en besogne : n'en déduisons pas qu'il s'agit d'un accident de parcours ni d'un habitus qui préfigurerait nos choix. Ce n'est peut-être qu'une expression (ni plus, ni moins) de cette "part riveraine du social» qui s'abandonne à sa "nervosité foncière» (Bordreuil, op.cit.), ou, pour ainsi dire, à sa labilité.

Quoique la "culture du projet» dise, les conflits d'urbanité ne nous abandonnent que rarement et la question fiduciaire y opère d'une façon déterminante ${ }^{7}$. La vulnérabilité expérientielle et la plasticité relationnelle qui participent à ces conflits et aux formes de l'urbanité qui s'y dégagent, nécessitent des architectures analytiques plus fines que celles de la domination et de la force, du retour de l'acteur et de la logique compassionnelle, du " tout stratégique » et des réseaux inter-objectifs (García Sánchez, 2012). Voila donc le défi actualisé : déployer des ressources critiques et politiques crédibles sans finir par conforter (surtout) les stéréotypes de la contestation, l'imagerie épique et le wishful thinking?

Peut-être qu'un flash-back to the roots permettrait de mieux viser l'horizon. Ne doit-on pas en grande partie à Pas à pas (Augoyard, 1979) et au Passant considérable (Joseph, 1984) le sursaut théorique qui avait permis à la recherche urbaine francophone, au début des années 1980, de modifier le curseur jusqu'alors monopolisé par les questions d'habitat? Par l'intermédiaire de ces ouvrages pionniers, la mobilité piétonne sensible, ordinaire et problématique avait pu enfin apparaître sérieusement en tant que vecteur central et éprouvant (mais affirmatif et inaliénable) de la fabrique des villes et de l'urbain. Aujourd'hui personne ne discute l'importance à y réfléchir de façon systématique et des bailleurs de fonds aussi divers que les régies de transport, les opérateurs de télécommunications et les agences de recherche n'hésitent plus à y investir pour déceler ses énigmes 8 . Mais cette place incitative, éclaircissante, critique, agissante et, tout simplement, politique, n'était guère acquise un quart de siècle avant.

\section{Ambiances et rencontres... là où on ne les attend pas}

Même si le «politiquement correct " nous dissuade de le faire savoir et de le valoriser explicitement, un congrès est enfin aussi, et surtout, un lieu de rencontres et, de ce fait, un espace-temps propice à l'entre-deux. Par sa nature composite, son inclinaison au jeu et sa culture fondamentalement sociable, l'entre-deux ne limite pas son agir associatif au politique ${ }^{9}$. C'est pourquoi celui qui cultive l'entre-deux peut s'affranchir sans regrets de la grammaire de la domination ou de la stratégie. On pourrait d'ailleurs mesurer la satisfaction que l'on retire d'un congrès à partir des rencontres de qualité qu'on y arrive à faire, à renouveler et/ou à perpétuer. Que cela soit avec des idées, des professionnels, des réseaux, des images, des communications orales, des lieux, des événements, des situations et/ou des perspectives, ces rencontres relèvent d'une expérience qui est, avant tout, singulière et interactionnelle. Ce qui veut dire aussi que dans un "bon congrès » (et, grâce aux soins organisationnels de l'équipe pilotée par Daniel Siret et Jean-Paul Thibaud, celui sur les ambiances à Montréal en a été un), les 
rencontres se font dans les conférences et les débats aussi bien que dans les pauses cafés, les sorties, les repas ou les couloirs au détour d'une séance.

Si le congrès de Grenoble avait placé la barre très (trop ?) haut, celui de Montréal a relevé le défi d'inciter les belles rencontres et de les aménager au long et à travers des contextes et des situations où les ambiances ont été tout sauf du luxe, du vent et de l'accessoire. Voici trois illustrations distinctes (par leur nature, leur forme et leur conséquence) de ce pari réussi.

$191 /$ Contrairement à ce qui est devenu «habituel» dans certains rassemblements savants, la séance consacrée aux posters sélectionnés par le comité scientifique n'était en rien mineure ni de second choix. Organisée selon le mode du " parcours commenté " ${ }^{10}$, menée par Eric Monin et ponctuée par des présentations / débats avec les réalisateurs devant leurs travaux, cela a été, me semble-t-il, une excellente occasion d'échange, formalisé certes. Pour autant, ce parcours n'a pas seulement «donné à voir» des travaux: il est devenu un espace-temps souple de rencontres multiponctuelles où l'essentiel ne s'est pas toujours (ni pour tous) passé au cœur de la scène (qui, de toute façon, au gré des présentations, changeait de place).

20 2/ Un directeur de laboratoire, un responsable d'organisme de tutelle et un invité privilégié par les circonstances prolongent la pause dans un couloir le temps d'une discussion à bâtons rompus sur le statut théorique des perspectives ouvertes par les travaux sur les ambiances. Aucune carte de présentation n'est mise en avant sauf la provenance disciplinaire (architecture pour les uns et sociologie pour l'autre). Aucune hiérarchie n'est défendue ni contestée à l'exception de celle qui émerge des épreuves de réalité auxquelles sont soumises les théories architecturales et urbaines. Et celles qui s'avèrent inégalement attentives aux enjeux techniques et socio-spatiaux se trouveront en bas de l'échelle.

21 3/ Lors de la " promenade d'ambiance » consacrée au corps en mouvement que MarieFrançoise Garcia a encadrée à Grenoble quatre ans auparavant, elle m'avait appris l'existence de la proprioception, sorte de sixième sens permettant de percevoir (inconsciemment ou pas) les mouvements du corps ${ }^{11}$. Je la revois à Montréal lors de la présentation dans le Congrès du court-métrage De Eau en Bas (co-réalisé en 2011 avec Cécile Regnault et Chantal Dugave), volet filmique d'un projet fondé sur un parcours chorégraphié qu'elle mène avec ses étudiants du Conservatoire National Supérieur de Musique et Danse de Lyon. Ils invitent le public à y participer de façon active, tout en se promenant en ville. Les fruits sont aussi riches que divers : une pratique du lieu qui se substitue au spectaculaire, l'oscillation de l'ambiance entre le festif et le comique... Et, pour autant, on en apprend sur la « pulsativité » des actions collectives, tout en voyant apparaître la danse « là où on ne l'attend pas » (García \& Regnault, 2012).

Si enfin l'ambiance peut n'être que de l'esthétique, cette dernière ne sortira pas indemne de cette rencontre séculaire, jamais inopinée, encore inachevée, toujours pertinente. Pour qualifier cet autre entre-deux qui surgit ainsi, l'Ecole de Medellín utiliserait volontiers le terme d'" esthétique expansive» (Montoya, 1999). Edifiée non sur la beauté mais sur les interstices, cette esthétique se préoccupe des affects tout autant que des percepts, incitant ainsi «le social " à s'échapper des scènes trop convenues. On le retrouvera néanmoins inséré dans une écologie matérielle (et parfois urbaine) qui ne sera plus simplement spatiale, ni strictement humaine ${ }^{12}$. L'entre-deux peut alors apparaître comme un chantier sensible de l'urbanité où le "sensible " prend une triple signification: boîte de résonance des troubles liés aux incertitudes du tout 
venant ${ }^{13}$, ambiance où sont considérés à leur juste valeur les éléments nourrissant l'expérience perceptive et lieu où sont investis les pulsions, les savoirs et les faire créatifs (García Sánchez, 2011). Ainsi, la part de soi que l'on risque d'égarer dans l'expérience de l'entre-deux ne nous rendra pas moins sensible à un apprentissage enrichissant, parce qu'éprouvant: celui de l'action qui se (pré)occupe des entours et qui ne fait sens qu'en devenant conjointe.

\section{BIBLIOGRAPHIE}

Augoyard, Jean-François. 1979. Pas à pas. Essai sur le cheminement quotidien en milieu urbain. Paris : Seuil.

Augoyard, Jean-François. 2011a. Faire une ambiance ? In : Augoyard, Jean-François (ed.). Faire une ambiance. Actes du colloque international, Grenoble, septembre 2008. Grenoble : Editions A la croisée. p. 17-35.

Augoyard, Jean-François (ed.). 2011b. Faire une ambiance. Actes du colloque international, Grenoble, septembre 2008. Grenoble : Editions A la croisée.

Battegay, Alain. 2011. Ambivalences de la citoyenneté. Migrations Société. vol. 23, n 136, p. 57-66.

Bonicco-Donato, Céline. 2012. Une lecture politique des ambiances urbaines. Entre hospitalité émancipatrice et stratégie disciplinaire. In : Thibaud Jean-Paul \& Siret Daniel (eds.). Ambiances in action / Ambiances en acte(s). Proceedings of the 2nd International congress on Ambiances, Actes du 2nd Congrès International sur les Ambiances. Grenoble : Réseau International Ambiances. p. 609-614.

Bordreuil, Jean-Samuel. 2007. Isaac Joseph : la politique depuis Goffman. In : Cefaï Daniel \& Saturno Carole (eds.). Itinéraires d'un pragmatiste. Autour d'Isaac Joseph. Paris : Economica. p. 101-134.

Breviglieri, Marc. 2012. L'espace habité que réclame l'assurance intime du pouvoir. Un essai d'approfondissement sociologique de l'anthropologie capacitaire de Paul Ricoeur. Études Ricouriennes / Ricœur Studies. vol. 3, n 1, p. 34-52.

Chelkoff, Grégoire. 1996. L'urbanité des sens : perceptions et conceptions des espaces publics urbains. Thèse de doctorat en urbanisme et aménagement. Grenoble : Université Pierre Mendès-France / IUG.

Chelkoff, Grégoire. 2012. L'ambiance sensible à l'architecture : paradoxes et empathies contemporaines ", In : Thibaud Jean-Paul \& Siret Daniel (eds.). Ambiances in action / Ambiances en acte(s). Proceedings of the 2nd International congress on Ambiances, Actes du 2nd Congrès International sur les Ambiances. Grenoble : Réseau International Ambiances. p. 27-32.

De Villanova, Roselyne. 2003. Architectures et cultures de l'entre-deux. Espaces et Sociétés. $\mathrm{n}^{\circ} 113-114$, p. 163-183.

De Villanova Roselyne \& Bonnin Philippe (eds.). 2006. Loges, concierges et gardiens, enquêtes en Europe. Paris : Ed. Créaphis. 
Desroches, Dominic. 2012. La fabrication des ambiances à partager. Le défi de la climatologie politique. In : Thibaud Jean-Paul \& Siret Daniel (eds.). Ambiances in action / Ambiances en acte(s). Proceedings of the 2nd International congress on Ambiances, Actes du 2nd Congrès International sur les Ambiances. Grenoble : Réseau International Ambiances. p. 141-144.

Diminescu, Dana. 2002. L'usage du téléphone portable par les migrants en situation précaire. Hommes et Migrations. $n^{\circ} 1240$, p. 66-79.

Diminescu Dana \& Pasquier Dominique (eds.). 2010. Les migrants connectés. T.I.C., mobilités et migrations. Réseaux. vol. 28, n 159.

Garcia Marie-Françoise \& Regnault Cécile. 2012. De Eau en Bas. La danse comme champs d'exploration des ambiances. In : Thibaud Jean-Paul \& Siret Daniel (eds.). Ambiances in action / Ambiances en acte(s). Proceedings of the 2nd International congress on Ambiances, Actes du 2nd Congrès International sur les Ambiances. Grenoble : Réseau International Ambiances. p. 111-116.

García Sánchez, Pedro J. 2012. Interactivité socio-spatiale et démocratie urbaine. Ce que la vulnérabilité de l'expérience peut (encore) nous apprendre. In : De Villanova Roselyne \& Duarte Christiane (eds.). Nouveaux regards sur l'habiter. Outils et méthodes, de l'architecture aux sciences sociales. Paris : Ed. Le Manuscrit. p. 223-243.

García Sánchez, Pedro J. 2011. Fronteras y umbrales de la urbanidad. Pensar el entre-dos urbano. In : Ribas Mateos Natalia (ed.). El Río Bravo Mediterraneo. Las regiones fronterizas en la época de la globalización. Barcelona : Bellaterra. p. 93-109.

Hollard, Arnaud. 2012. Renouveler le renouvellement urbain? Les ambiances face aux enjeux de la rénovation des grands ensembles. In : Thibaud Jean-Paul \& Siret Daniel (eds.). Ambiances in action / Ambiances en acte(s). Proceedings of the 2nd International congress on Ambiances, Actes du 2nd Congrès International sur les Ambiances. Grenoble : Réseau International Ambiances. p. 377-382.

James, William. 1898. Philosophical Conceptions and Practical Results [en ligne]. University Chronicle. vol. I ${ }^{\circ}$ 4. Mis en ligne le 31.07.2006, consulté le 10.10.2013. Url : http:// www.archive.org/stream/philosophicalcon00jameuoft/philosophicalcon00jameuoft_djvu.txt.

Joseph, Isaac. 1984. Le passant considérable. Essai sur la dispersion de l'espace public. Paris : Librairie des Méridiens.

Montoya, Jairo. 1999. Desarticulaciones urbanas. In Montoya Jairo \& Xibillé Jaime (eds.). Memorias y percepciones del paisaje urbano. Medellin : Ed. Universidad Pontificia Bolivariana. p. 5-30.

Pecqueux, Anthony. 2012. Politique des ambiances. Que faire du fond inextricablement politique des ambiances ? In : Thibaud Jean-Paul \& Siret Daniel (eds.). Ambiances in action / Ambiances en acte(s). Proceedings of the 2nd International congress on Ambiances, Actes du 2nd Congrès International sur les Ambiances. Grenoble : Réseau International Ambiances. p. 145-151.

Simmel, Georg. 1980. Sociologie de la sociabilité. Urbi. III, p. 109-114.

Simonnot, Nathalie. 2012. Le paradoxe de la patrimonialisation des ambiances. In : Thibaud JeanPaul \& Siret Daniel (eds.). Ambiances in action / Ambiances en acte(s). Proceedings of the 2nd International congress on Ambiances, Actes du 2nd Congrès International sur les Ambiances. Grenoble : Réseau International Ambiances. p. 33-38.

Thévenot, Laurent. 2006. L'action au pluriel. Sociologie des régimes d'engagement. Paris : La Découverte.

Thibaud, Jean-Paul. 2001. La méthode des parcours commentés. In : Grosjean Michèle \& Thibaud Jean-Paul (dir.). L'espace urbain en méthodes. Marseille : Ed. Parenthèses. p. 79-99. 
Thibaud Jean-Paul \& Siret Daniel (eds.). 2012. Ambiances in action / Ambiances en acte(s). Proceedings of the 2nd International congress on Ambiances, Actes du 2nd Congrès International sur les Ambiances. Grenoble : Réseau International Ambiances.

Thomas, Rachel. 2012. Les perspectives critiques de la notion d'ambiance. In : Thibaud Jean-Paul \& Siret Daniel (eds.). Ambiances in action / Ambiances en acte(s). Proceedings of the 2nd International congress on Ambiances, Actes du 2nd Congrès International sur les Ambiances. Grenoble : Réseau International Ambiances. p. 45-51.

Zardini, Mirko. 2012. Toward a sensorial urbanism. In : Thibaud Jean-Paul \& Siret Daniel (eds.). Ambiances in action / Ambiances en acte(s). Proceedings of the 2nd International congress on Ambiances, Actes $d u$ 2nd Congrès International sur les Ambiances. Grenoble : Réseau International Ambiances. p. 19-26.

Wirth, Louis. 1979 [1938]. Le phénomène urbain comme mode de vie. In : Grafmeyer Yves \& Joseph Isaac (eds.). L'Ecole de Chicago. Naissance de l'écologie urbaine. Paris : Editions du champ urbain. p. 255-281.

\section{NOTES}

1. Depuis l'époque de Robert Park et William Thomas, en passant par Howard Becker, Erving Goffman, Everett Hughes ou Anselm Strauss, et jusqu'à Michael Burawoy ou Joseph Gusfield, au moins trois générations d'« Ecole de Chicago » se sont succédées, en accordant toujours une place déterminante aux dimensions sensibles du travail ethnographique.

2. L'expression « On connaît l'arbre à ses fruits, non à ses racines » résume ce propos de William James. Ainsi, « Pour atteindre une parfaite clarté dans nos pensées sur un objet, il suffit de tenir compte des effets pratiques concevables que cet objet peut impliquer - quelles sensations nous pouvons en espérer? quelles réactions faut-il préparer?" ("To attain perfect clearness in our thoughts of an object, then, we need only consider what effects of a conceivably practical kind the object may involve - what sensations we are to expect from it, and what reactions we must prepare", James, 1898).

3. Toujours autour des épreuves propres à la contemporanéité du sujet, G. Chelkoff (2012) a fait part à Montréal de son questionnement actuel à propos des "paradoxes d'ambiance »; N. Simonnot (2012) porte la même interrogation à propos de la patrimonialisation.

4. Signalons qu'il s'agissait des conférences publiques organisées par le Centre Canadien d'Architecture (où a eu lieu le congrès), conférences dont le sujet permettait l'intégration au programme du $2^{\text {nd }}$ Congrès International sur les Ambiances.

5. Ce terme pouvant qualifier, à la fois (ou de façon distincte), une temporalité relevant de l'ordinaire et une socialité fondée sur le partage du même.

6. Parmi les démarches qui prolongent la réflexivité critique et la mise en politique du sensible dans une visée pluraliste, je retiens en particulier les suivantes: (1) la «politisation» de la microsociologie que Jean-Samuel Bordreuil souligne dans l'interprétation josephienne de Goffman (op.cit.); (2) l'anthropologie capacitaire qui interroge l'habiter depuis la philosophie ricoeurienne (Breviglieri, 2012); (3) la sociologie des régimes d'engament mise à point par Laurent Thévenot (2006) et (4) la sociologie des migrations intéressée par les "ambivalences » d'une citoyenneté devant composer avec les «cultures de carrefour» qui reformulent le cosmopolitisme contemporain (Battegay, 2011).

7. «Tout discrédit ne fait pas politique. Pourtant les moments proprement politiques ne s'amorcent jamais que dans et par la relève publique d'un discrédit » (Bordreuil, op.cit.). 
8. C'est dans cette ligne que, par exemple, s'inscrit aujourd'hui le travail développé au sein de MUSE, acronyme du projet de recherche «Les énigmes sensibles des mobilités urbaines contemporaines". Réunissant des chercheurs français, anglais, espagnols, brésiliens et vénézuéliens, ce projet avait été retenu par l'Agence Nationale de la Recherche (ANR) de France dans l'appel de son programme "Espace et territoire : les énigmes spatiales de la vie en société » en 2010. Cf. également les travaux de Dana Diminescu (Diminescu, 2002 ; Diminescu \& Pasquier, 2010) autour de la figure du « migrant connecté ».

9. « On pourrait parler d'une impulsion à la sociabilité chez l'homme (...) Certes, c'est en fonction d'intérêts et de besoins particuliers que les hommes s'unissent dans des associations économiques, dans des pactes de sang, dans des sociétés de cultes et des bandes des voleurs. Mais en-deçà et au-delà de leur contenu particulier, toutes ces associations s'accompagnent d'un attrait, d'un consentement qui tient au seul fait d'être associé à d'autres (...) La sociabilité est la forme ludique des forces éthiques de la société concrète » (Simmel, 1980).

10. A ce propos, on ne remerciera jamais assez les collègues du CRESSON et la mise au point méthodologique effectuée par Jean-Paul Thibaud (2001). Cette technique d'enquête féconde par la mise en contexte des situations a réussi ainsi à s'introduire dans la «boite à outils » dont aujourd'hui se servent non seulement les chercheurs en sciences sociales travaillant sur l'espace, mais aussi les collectivités territoriales, la "gestion urbaine de proximité » (GUP) et d'autres dispositifs de travail socio-spatial.

11. La kinesthésie aurait une signification proche, à la différence que celle-ci exclut le sens de l'équilibre.

12. A cet égard, les travaux de Roselyne De Villanova (De Villanova, 2003 ; De Villanova \& Bonnin, 2006) sur l'entre-deux architectural mis à l'œuvre par les migrants bâtisseurs ou sur l'habiter auquel contribuent les loges et les gardiens, témoignent d'une quête singulière qui a permis de situer l'enquête interculturelle au croisement de l'urbanisme et de l'urbanité.

13. Ceci incite par ailleurs à penser que, au-delà du stigmate et des impropriétés éventuelles, l'utilisation en français du qualificatif "sensible» pour connoter les quartiers en difficulté, mériterait d'être considérée avec des égards davantage analytiques.

\section{AUTEUR}

\section{PEDRO JOSÉ GARCÍA SÁNCHEZ}

Sociologue-ethnographe, maître de conférences en sociologie à l'Université Paris Ouest Nanterre - La Défense (où il est co-responsable du « Parcours en sociologie et anthropologie urbaines ») et chercheur dans le laboratoire Mosaïques/LAVUE UMR CNRS 7218. S'intéressant aux registres perceptifs, cognitifs et performatifs de l'interaction, il a développé une approche pragmatiste sur les conflits d'urbanité et sur l'écologie de l'ordre public. Des terrains contrastés dans des villes latino-américaines, africaines et de la banlieue parisienne l'ont amené à esquisser une sociologie du trouble urbain et de la vulnérabilité dépliée. Il analyse ainsi les formes élémentaires et sensibles de la vie citadine et la démocratie urbaine à l'œuvre.

pjgarcia@u-paris10.fr

http://www.laboratoire-mosaiques.fr/_PEDRO-JOSE-GARCIA-SANCHEZ_html 